\title{
NUMERICAL COMPUTATION OF INFRAGRAVITY WAVE DYNAMICS AND VELOCITY PROFILES USING A FULLY NONLINEAR BOUSSINESQ MODEL
}

\author{
R. Cienfuegos ${ }^{1}$, L. Duarte ${ }^{*}$, L. Suarez ${ }^{*}$ and P. A. Catalán ${ }^{2}$
}

\begin{abstract}
We present experimental and numerical analysis of nonlinear processes responsible for generating infragravity waves in the nearshore. We provide new experimental data on random wave propagation and associated velocity profiles in the shoaling and surf zones of a very mild slope beach. We analyze low frequency wave generation mechanisms and dynamics along the beach and examine in detail the ability of the fully nonlinear Boussinesq- type model SERR1D (Cienfuegos et al., 2010) to reproduce the complex dynamics of high frequency wave propagation and energy transfer mechanisms that enhance infragravity wave generation in the laboratory.
\end{abstract}

\section{INTRODUCTION}

Surf zone processes and beach profile response are influenced by rich and complex nonlinear wave interactions involving a wide range of spatial and temporal scales. In particular, nonlinear propagation of incident wind-generated waves $(f \sim 0.05-1 \mathrm{~Hz})$ may produce bound low frequency waves $(f \sim 0.001-0.05 \mathrm{~Hz})$ that can eventually be released as free long waves and even be reflected and/or refractively trapped near the shoreline. As a consequence, the resulting cross-shore pattern of the velocity field, residual currents and sediment transport will be strongly affected by oscillatory motions occurring at a broad range of scales.

Numerical modeling of long wave generation by transient groups in deep and intermediate water depths is well established and in good agreement with laboratory data (see for instance Johannessen and Swan, 2003). Long-wave propagation in shallow water has been addressed using non-dispersive models based on the shallow water equations (Hibberd and Peregrine, 1979), and some aspects of the surf beat generation mechanism have been successfully reproduced by forcing these models with radiation stress gradients of wave heights across the group (List, 1992). However, examples of numerical modeling of the complete infragravity wave generation process and dynamics from realistic random wave fields propagating over beach profiles are scarce. Important efforts in that direction have been accomplished by Madsen et al. (1997) applying a Boussinesq-type model to a bichromatic wave data set. Similar applications of a Boussinesq model to swash oscillations and infragravity motions are described in Karunarathna et al. (2005). Nevertheless, to the authors' knowledge, most of the applications of Boussinesq modeling to infragravity wave generation has been reported for bichromatic wave fields and using weakly nonlinear versions of model equations.

The objective of the present article is twofold. First, we aim at providing new experimental data of random wave propagation and associated velocity profiles in the shoaling and surf zones, including low frequency wave (LFW) generation and dynamics over a very mild slope beach. Second, we examine in detail the ability of the fully nonlinear Boussinesq-type model SERR1D (Cienfuegos et al., 2010) to reproduce the complex dynamics of high frequency wave propagation; and the energy transfer mechanisms that enhance infragravity wave generation in the laboratory experiment. SERR1D includes parameterizations for breaking and swash processes that have been previously validated on regular waves.

\section{EXPERIMENTS AND NUMERICAL MODELING}

\section{Description of laboratory experiments}

Wave experiments were conducted in the laboratory wave flume of the Instituto Nacional de Hidráulica (INH) located in Peñaflor, near Santiago of Chile. The flume is $70 \mathrm{~m}$ long and has a square cross section with side length of $1.5 \mathrm{~m}$. It has concrete bottom and side walls and was prepared with an impermeable smooth concrete plane beach of $1 / 80$ slope $(x=0 \mathrm{~m}$ at the toe of the slope, positive shorewards, see Figure 1). The flume is equipped with a hydraulically driven piston-type wave paddle from the Danish Hydraulic Institute (DHI) with second-order wave generation (Schäffer, 1996). Back reflection at the wave paddle is minimized by active absorption through DHI-AWACS system (Active

\footnotetext{
${ }^{1}$ Departamento de Ingeniería Hidráulica y Ambiental, Pontificia Universidad Católica de Chile, Vicuña Mackenna 4860, casilla 306, correo 221, Santiago,Chile. Email : racienfu@ing.puc.cl

${ }^{2}$ Departamento de Obras Civiles, Universidad Técnica Federico Santa María, Av España 1680 , Casilla 110-V, Valparaíso, Chile. Email : patricio.catalan@usm.cl
} 
Wave Absorption Control System). A large number of experiments were run, using a single 25-minute wave signal time series derived from a JONSWAP type spectrum with a significant wave height $H$ $=18 \mathrm{~cm}$, a peak frequecy $f_{p}=0.25 \mathrm{~Hz}$; and peak-enhancement factor $\gamma=3.3$. The still water depth at the wave maker was fixed at $h_{0}=52 \mathrm{~cm}$. Measurements of the free surface displacements were performed all over its length at high spatial resolution $(0.2 \mathrm{~m}$ to $1 \mathrm{~m})$ using resistive probes. Velocity profiles were recorded at five locations within the shoaling and surf zones using a Sontek MicroADV. Repeatability of the experiments was verified by keeping a fixed probe at a distance of $2 \mathrm{~m}$ from the wave-maker and performing redundant measurements at several other locations. In Figure 2 we show that the measured wave height histogram is in agreement with the theoretical Rayleigh distribution $2 \mathrm{~m}$ away from the wave paddle.

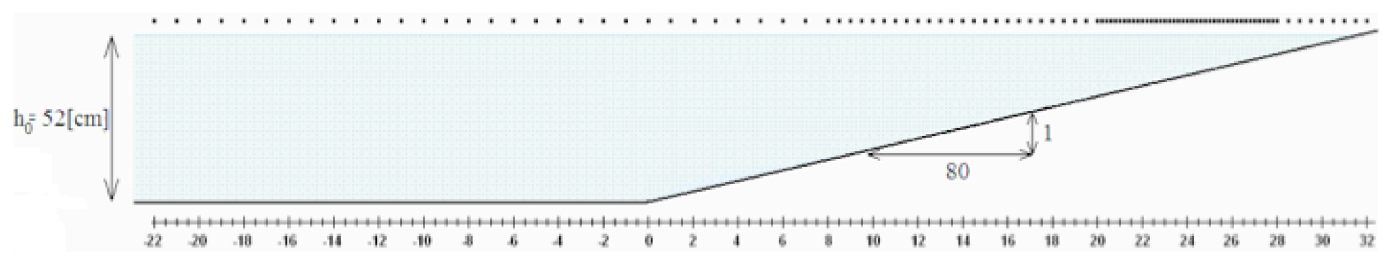

Figure 1: Sketch of the experimental wave tank at the Instituto Nacional de Hidráulica and wave gauge locations.

Velocity and free surface measurements were acquired at $20 \mathrm{~Hz}$, and a simple low-pass filter was used to separate orbital motions from turbulence in velocity time series (Nadaoka et al., 1989). The cut-off frequency was chosen at $3 \mathrm{~Hz}$, i.e., nearly twelve times the peak frequency of the JONSWAP wave spectrum.

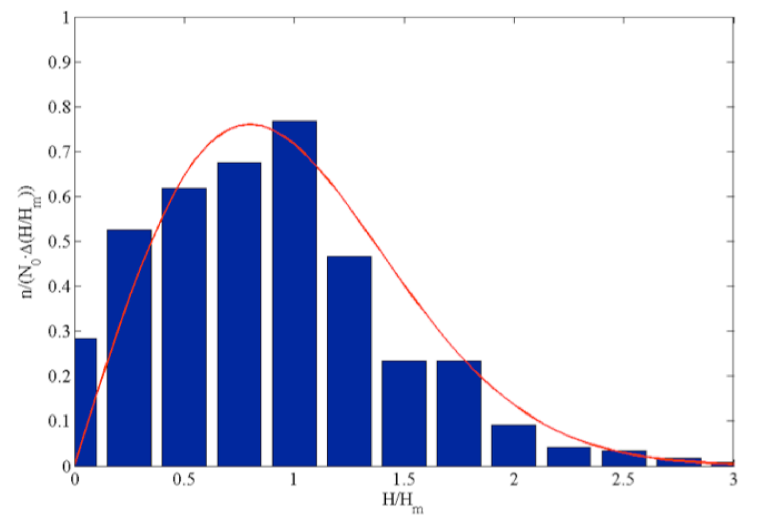

Figure 2: Normalized histogram of wave heights measured at a distance of $2 \mathrm{~m}$ from the wave paddle. Theoretical Rayleigh distribution in solid line. $H$ is the wave height and ${ }_{m}$ is the mean wave height. 


\section{Overview of SERR1D}

Numerical computations are conducted using the 4th-order finite volume Boussinesq model SERR1D (Cienfuegos et al., 2010). After inclusion of wave-breaking energy dissipation, the mass and momentum conservation equations can be written in the following form

$$
\begin{aligned}
h_{t}+F_{x} & =D_{1}, \\
q_{t}+G_{x} & =\frac{1}{h} D_{2}-S,
\end{aligned}
$$

where $e$ is the water density, $h$ is the water depth, and $D_{1}, D_{2}$ represent breaking-induced functions. Here, variables $x$ and $t$ denote fixed space and time coordinates and sub-indices represent partial derivatives. Remaining functions and variables are defined as,

$$
\begin{gathered}
\left.q=\left[1+\left(h_{x}+\xi_{x}\right) \xi_{x}+\frac{1}{2} h \xi_{x x}\right]\right) u-\frac{1}{3 h}\left(h^{3} u_{x}\right)_{x}-\alpha \xi^{2} u_{x x}, \\
F=h u, \\
G=q u+g(h+\xi)-\frac{1}{2} u^{2}\left(1+\xi_{x}^{2}\right)+\left(\xi_{x} u-\frac{1}{2} h u_{x}\right) h u_{x} \\
-\alpha \xi^{2}\left[u_{x}^{2}+u u_{x x}+g(h+\xi)_{x x}\right], \\
S=2 \alpha \xi \xi_{x}\left[u u_{x}+g(h+\xi)_{x}\right],
\end{gathered}
$$

where $u$ is the depth-averaged horizontal velocity; $g$ is the acceleration of gravity, and $z=\xi$ is the vertical coordinate of the bottom measured from the still water depth $(z$ is positive upwards and $d=|\xi|$ is the still water depth). $\alpha$ is a dispersion correction parameter set to $\alpha=1 / 15$ to produce a Padé approximant to the linear Stokes dispersion relation (Cienfuegos et al., 2007). Details on the wave breaking parameterization and implementation can be found in Cienfuegos et al. (2010). Default parameter values will be adopted in the remaining of this article.

\section{RESULTS}

For the numerical computations, we have forced the model using measured free surface time series at the wave gauge located at a distance of $2 \mathrm{~m}$ from the wave paddle. Since one of the objectives of the present article is to assess the ability of the Boussinesq model to generate LFW from a narrow banded JONSWAP spectrum, we have high-pass filtered the measured signal to remove the infragravity wave energy before using it as boundary condition. In Figure 3 we compare the measured density variance spectrum from the experiment to the high-pass filtered signal used as forcing for the numerical model. It can be seen that in the experiment, there is a non-negligible energy content in the infragravity band frequencies $(f<0.01 \mathrm{~Hz})$ and that the filtering technique succeeds in removing most of this energy without affecting the narrow banded JONSWAP spectrum. The absorbing-generating boundary condition described in Cienfuegos et al. (2007) and Mignot and Cienfuegos (2009) is implemented at the seaward boundary, while the extrapolation technique of Lynett et al. (2002) is used to model the moving shoreline boundary condition. 


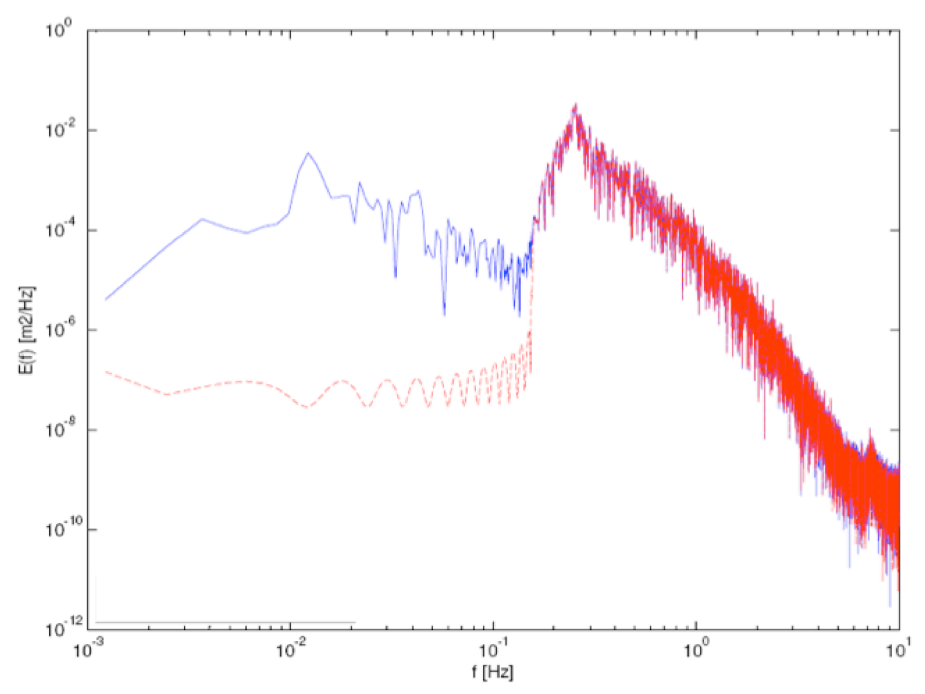

Figure 3: Density variance spectrum of measured free surface at a distance of $2 \mathrm{~m}$ from the wave paddle (solid blue) and high-pass filtered signal used as boundary condition for the numerical model (dash red).

\section{Wave energy evolution along the beach}

In Figure 4 we compare the root mean square wave heights $\left(H_{r m s}\right)$ and mean water level (set-up) between measurements and model throughout the wave tank. A reasonable agreement if found in both quantities although the numerical model appears to underestimate energy dissipation in the surf zone by overpredicting wave height values.
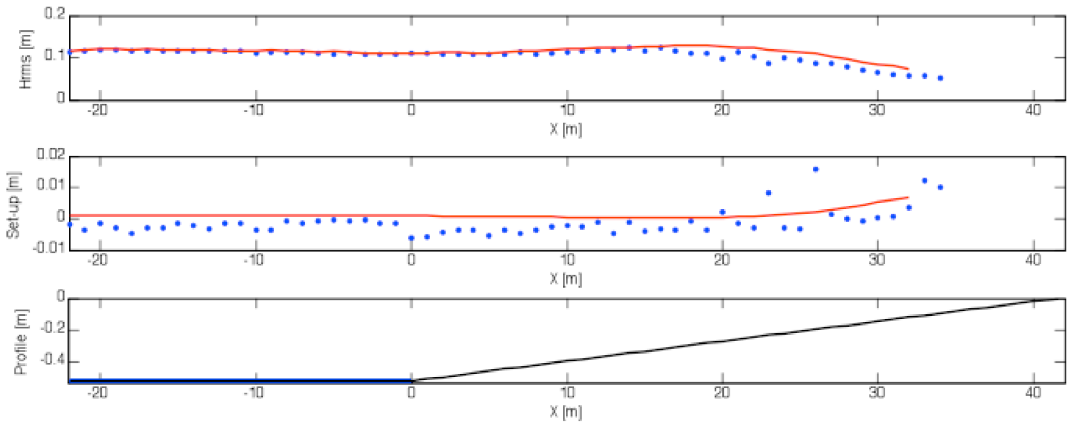

Figure 4: Spatial evolution of root mean square wave heights $\left(H_{r m s}\right)$ and mean water level (set-up) along the beach. Measured quantities are in symbols, and computed results appear in red plain lines. Upper panel : $H_{r m s}$; middle pannel : set-up; lower panel : bathymetric configuration.

The spectral energy evolution of measured and computed free surface time series is presented in Figure 5. It can be seen that the SERR1D is able to accurately reproduce the wave energy evolution in the JONSWAP frequency band over most of the beach profile. Indeed, nonlinear energy transfers from the peak frequency to higher harmonics is very well captured and in agreement with measurements. Moreover, energy dissipation by breaking is correctly predicted but slightly under estimated in the inner surf zone, consistent with the mentioned over prediction of wave heights. 

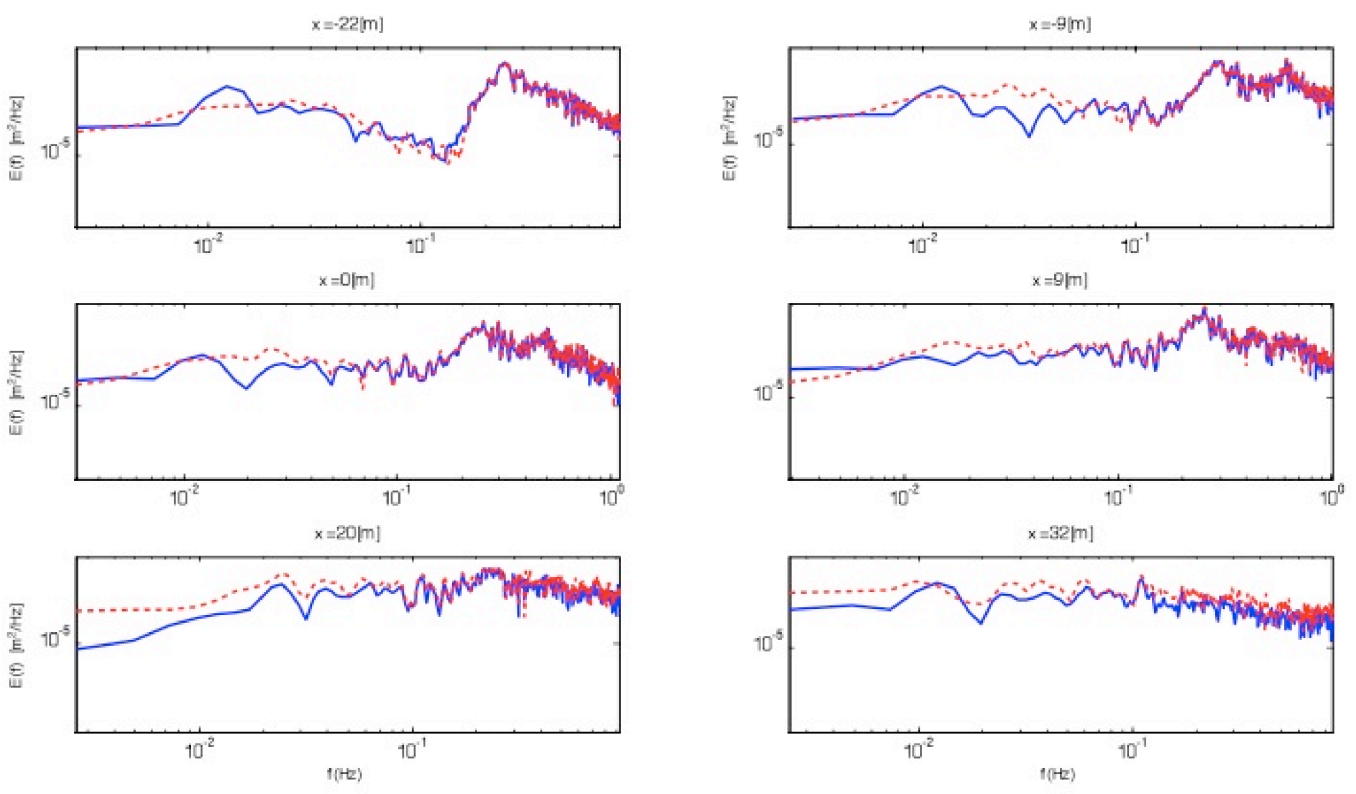

Figure 5: Spatial evolution of the density variance spectrum of measured (blue plain lines) and computed (dash red lines) free surface time series at several wave gauges.

Similarly, the numerical model succeeds in reproducing energy transfer to the infragravity band. Nevertheless, there are some discrepancies between observed and computed peak frequencies of the LFW that might be attributed to the different type of seaward boundary conditions used in the physical experiment and in the numerical wave tank. In the numerical model, an effective absorbing-generating boundary condition is employed, while in the laboratory, some LFW energy is reflected back to the wave tank at the paddle. The latter is responsible for creating quasi-standing waves in the wave tank which might amplify the amplitude of wave frequencies close to resonant modes (see below). It is worth noting that computed peak frequencies in the infragravity band are in close agreement with measurements at station $x=20 \mathrm{~m}$ and $x=32 \mathrm{~m}$, evidencing some degree of natural resonance of LFW in the upper part of the beach.

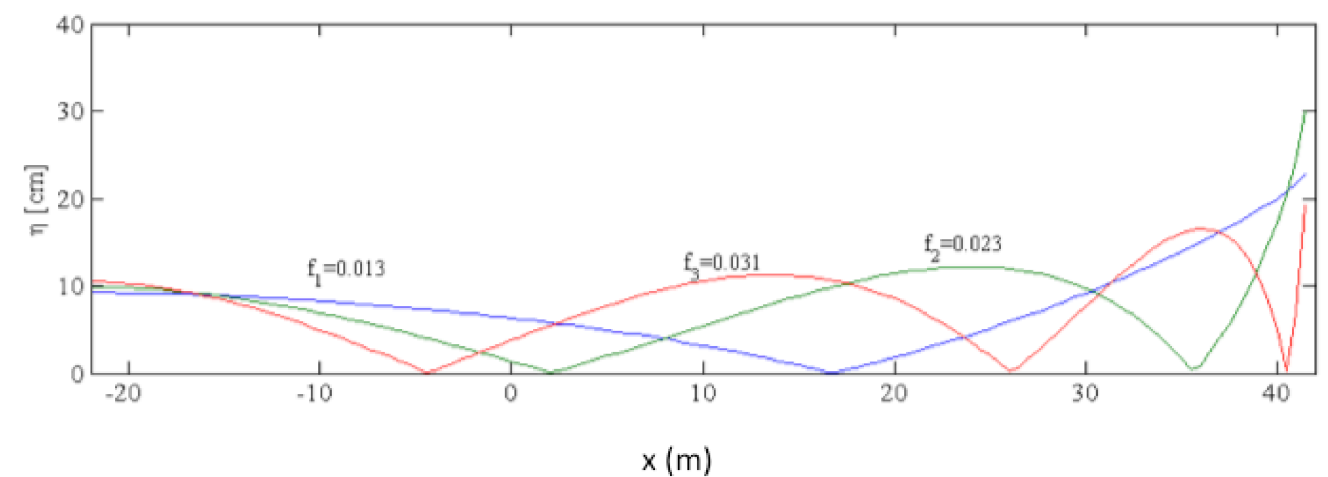

Figure 6: Spatial structure of the principal modes estimated from a linear normal mode analysis of the wave tank geometry. Blue : first mode; green : second mode; red : third mode.

A linear modal analysis has been performed considering the geometry of the wave tank bathymetry. The first three modes are depicted in Figure 6. The charachteristic spatial structure of standing waves is observed with the formation of nodes and anti-nodes for the main frequencies $f_{1}=0.013 \mathrm{~Hz}, f_{2}=0.023 \mathrm{~Hz}$ and $f_{3}=0.031 \mathrm{~Hz}$. These modes appear as peaks in the density variance spectra of measured free surfaces in Figure 5. 


\section{Low frequency wave generation and dynamics}

It has been previously postulated that energy transfer to the infragravity band is produced by two principal mechanisms : i) radiation stress variations through the slow modulation of wave height across the group (Biésel, 1952; Longuet-Higgins and Stewart, 1962), and ii) long-wave forcing by the oscillation of the breakpoint location (surf beat) (Symonds et al., 1982). The spectral evolution of wave energy along the tank presented in the previous subsection showed an important energy transfer from the narrow banded JONSWAP spectrum to low frequency waves. Next, we attempt to identify the generating mechanism and propagation dynamics of the resulting long waves, by means of a crosscorrelation analysis. This technique has been previously employed in the same context by Janssen et al. (2003) and Baldock (2006), among others.

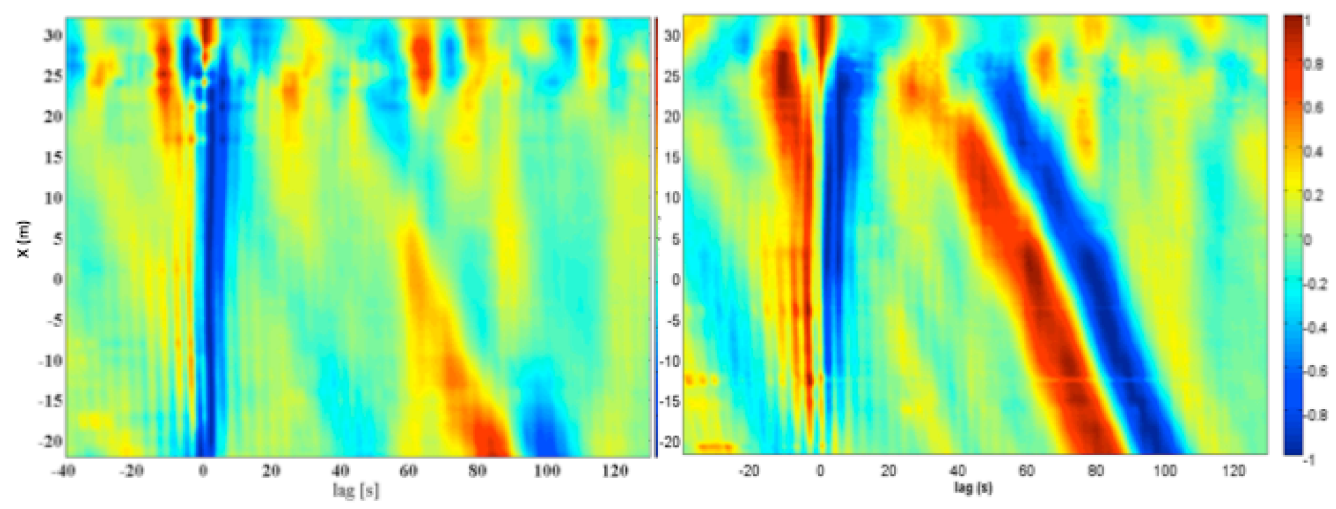

Figure 7: Cross-correlation maps between the envelope of high-frequency waves and low frequency waves (low pass-filtered at $f_{c}=0.01 \mathrm{~Hz}$ ) at each location. Left panel : experiments; right panel : numerical modeling.
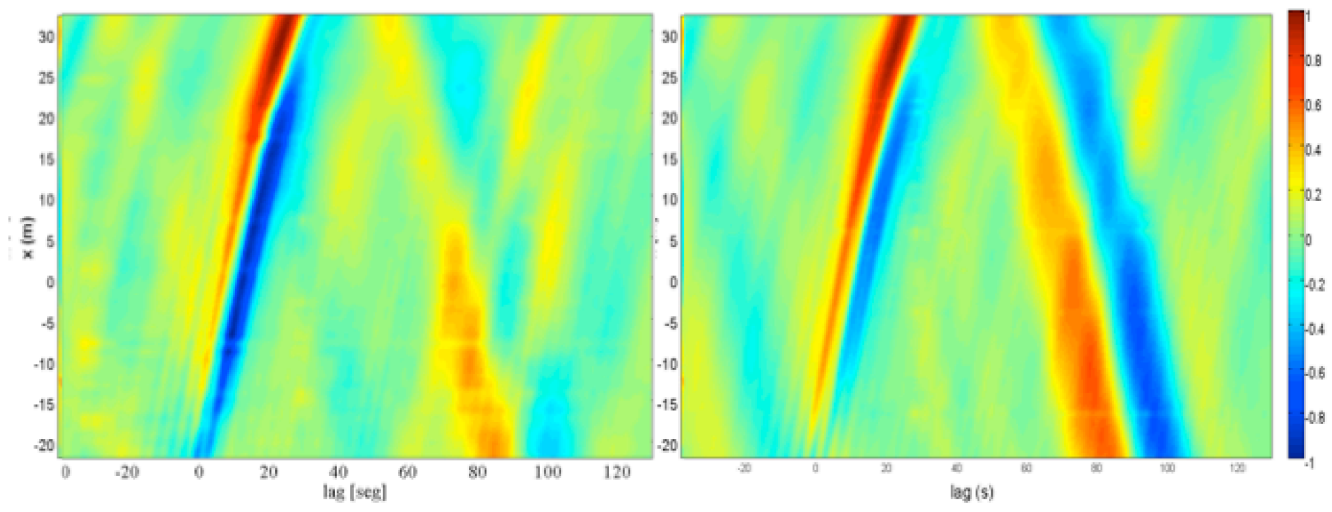

Figure 8: Cross-correlation maps between the envelope of high-frequency waves at the first wave gauge and low frequency waves (low pass-filtered at $f_{c}=0.01 \mathrm{~Hz}$ ) at each location. Left panel : experiments; right panel : numerical modeling.

In Figure 7 cross-correlation maps between the envelope of high-frequency waves and low frequency waves low pass-filtered at $f_{c}=0.01 \mathrm{~Hz}$ are presented. The cross-correlation is computed at each location in order to detect temporal lags and to identify bound waves. It is seen that there is an evident negative correlation with negligible phase lag in the shoaling zone up to $x=20 \mathrm{~m}$. This is the typical signature of a bound long wave propagating at the speed of the group. In the surf zone, a positive correlation appears, that leads the phase of the group. This may evidence a dynamic set-up mechanism of infragravity wave generation. There is a reasonable agreement in the spatio-temporal structure of LFW for measured and computed free surfaces, even if the correlation is stronger in the numerical computations. Computed results show that positive and negative correlations appear in the shoaling zone, with a positively correlated wave ahead of the group. It is worth noting that the numerical model has been forced without any energy in the infragravity band and without a forced bound wave. The 
latter might explain the observed differences between numerical and experimental results. In addition, long wave reflection at the beach can be indentified as well. This is clearly observed in the numerical results where two main long waves seem to be released freely seaward.

In Figure 8, cross-correlation maps between the envelope of high-frequency waves at the first wave gauge and low frequency waves at each location are depicted. This type of representation is useful for identifying the propagation velocity of the long waves. Again, two main infragravity waves are observed, one positively correlated ahead of the group, and one negatively correlated traveling at the group speed. The experimental conditions seem to enhance the generation of both LFW, from dynamic set-up and second order group modulation. It is interesting to note, that whereas the strength of the negatively correlated wave seems to decrease in the surf zone, the positively correlated one dominates there. Both LFW are partially reflected after the runup, and this is more evident in the numerical computation where bed shear stresses might be under estimated in the swash zone.

\section{Velocity statistics}

In this last section, we show results on several important statistic quantities related to velocity profiles. One of the advantages of using a phase-resolving numerical model is that it provides high-order moments of near bed velocity time series which are important for predicting sediment transport (see for instance Drake and Calantoni, 2001; Hoefel and Elgar, 2003).
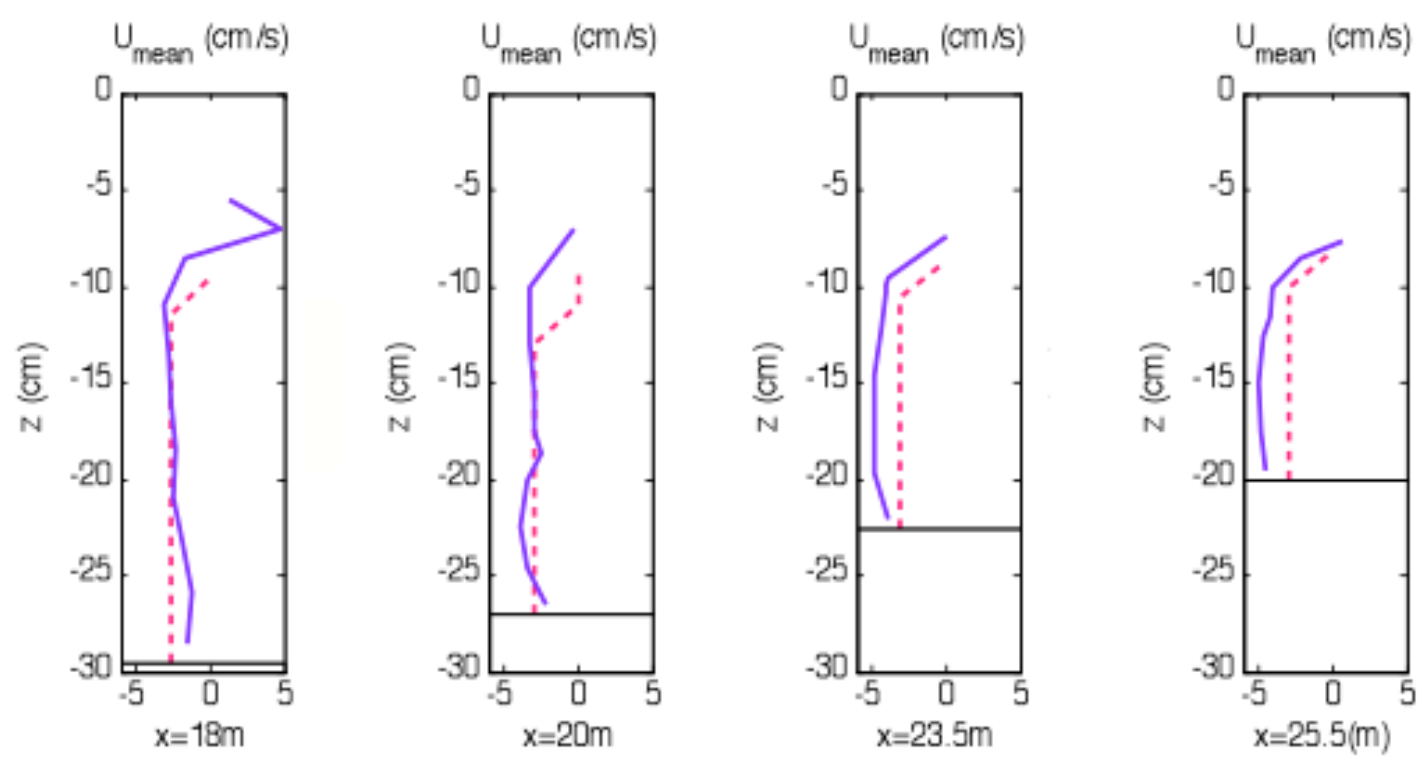

Figure 9: Mean velocity profiles averaged over the whole time series and for different verticals. Blue plain lines : experiments; dash red lines : numerical computation.

Mean currents, averaged over the whole duration of the experiment are presented in Figure 9, where numerical and experimental profiles are compared at multiple locations. It is seen that in the shoaling zone $(x \leq 20 \mathrm{~m})$, the agreement is fairly good, but inside the surf zone, there is an under prediction of the mean current by the model. These differences might be attributed to the breaking parameterization since $H_{r m s}$ values are over estimated in the surf zone according to results presented in Figure 4. Recalibrating the breaking model in order to get better agreement in the surf zone might also improve the estimation of mean currents since onshore velocities are over predicted in the numerical model due to the over estimation of wave heights in the inner surf zone. 

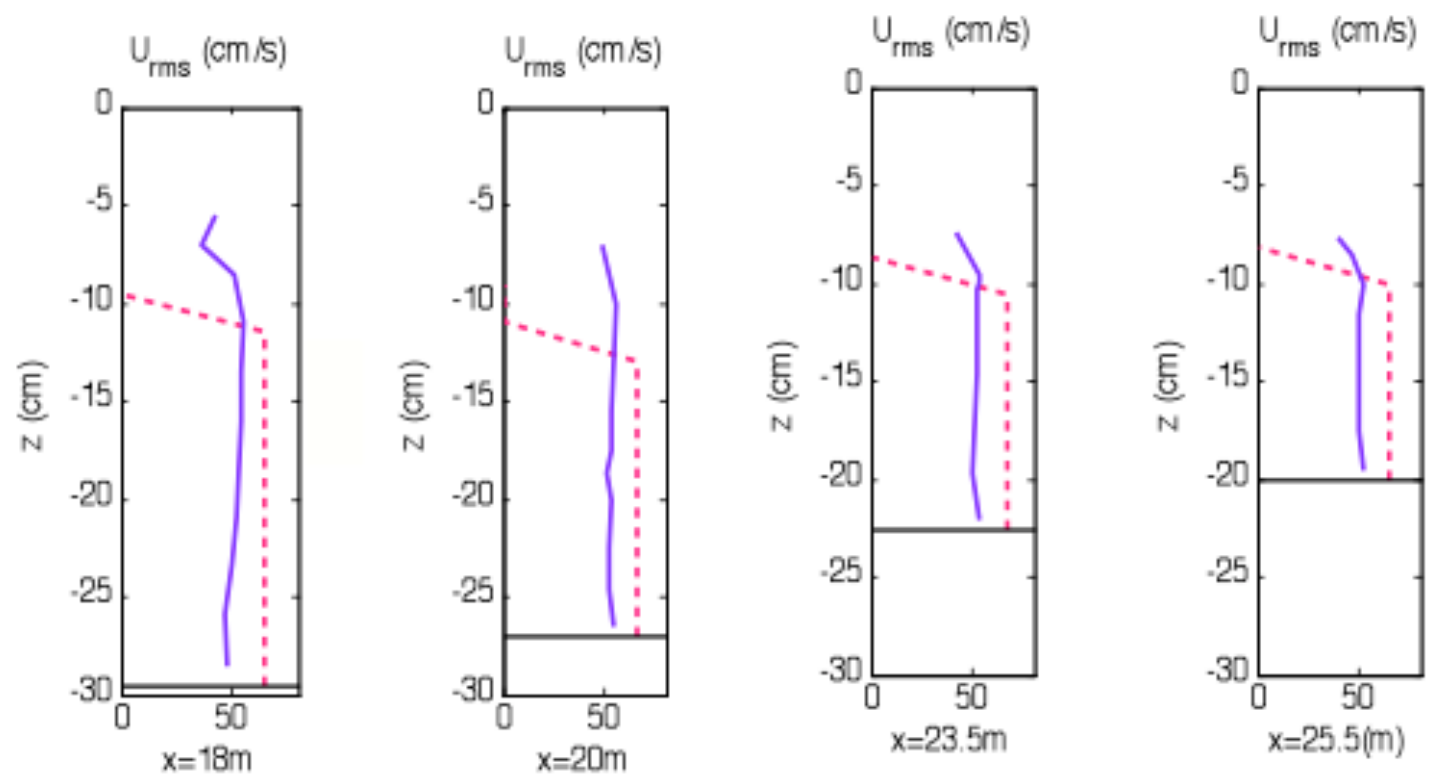

Figure 10: Root mean square (rms) velocity profiles averaged over the whole time series and for different verticals. Blue plain lines : experiments; dash red lines : numerical computation.
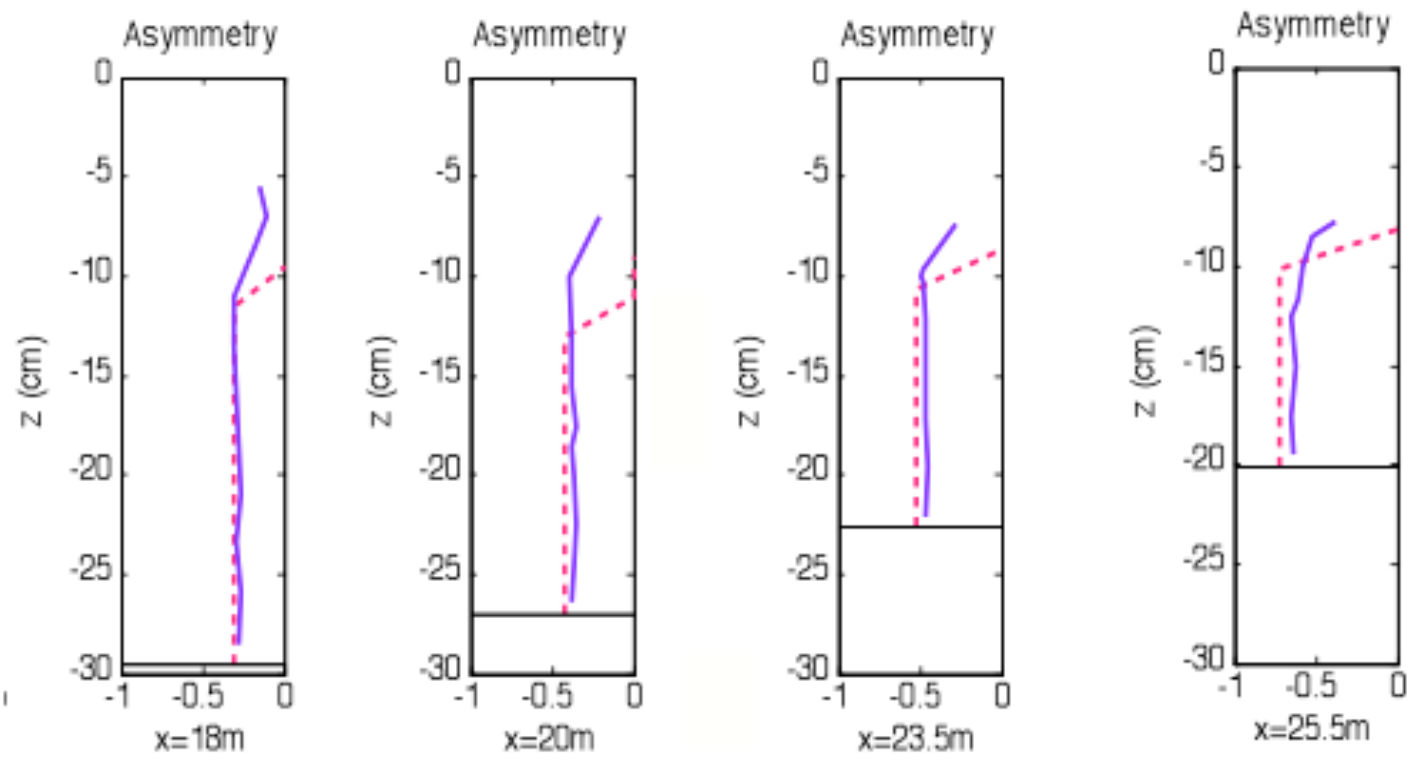

Figure 11: Left-right asymmetry of velocity profiles computed over the whole time series and for different verticals. Blue plain lines : experiments; dash red lines : numerical computation.

The over estimation of velocities in the shoreward direction is confirmed in Figure 10 where measured and computed root mean square velocities are depicted. There is a shift in the estimation of the amplitude of orbital velocities that should probably be associated to the over prediction of wave heights in the inner surf zone. Nevertheless, the vertical asymmetry (left-right asymmetry) of velocity time series, which is a key parameter for sediment transport prediction (Drake and Calantoni, 2001; Hoefel and Elgar, 2003), is very well computed by SERR1D according to results presented in Figure 11. The latter confirms some of the improvements achieved with the breaking wave parameterization introduced by Cienfuegos et al. (2010). 


\section{CONCLUSIONS}

In the present work, we have presented a new set of detailed free surface and velocity measurements of random wave propagation over a very mild slope beach. The investigated experimental conditions are highly demanding for nonlinear phase-resolving numerical models since a complex pattern of energy transfer from the primary narrow banded JONSWAP spectrum to the infragravity band is observed. Numerical predictions of infragravity wave generation and release were performed with SERR1D, a fully nonlinear and weakly dispersive Boussinesq-type model for non-breaking and breaking waves. These are in good agreement with experimental data. Nevertheless, some discrepancies are observed which seem to be related to an under prediction of wave breaking energy dissipation in the inner surf zone. Other differences might be attributed to the lack of bottom friction in the numerical model (particularly in the swash zone) and to the use of a filtered free surface signal as seaward boundary condition, since it does not consider forced bound waves associated to the incident groups.

Velocity profile statistics inside the surf zone are reasonably predicted by the numerical model but again, differences may be attributed to an underestimation of wave energy dissipation by the breaking parameterization (root mean square velocities are over predicted and mean currents are under predicted in the inner surf zone). SERR1D provides reasonable estimates for velocity moments and fluid acceleration under realistic conditions but the breaking model needs to be further improved considering a re-calibration on velocity and free surface measurements. Nevertheless, computed left-right asymmetry velocities, which appears to be a key parameter for sediment transport prediction, is in excellent agreement with measured values.

\section{ACKNOWLEDGMENTS}

This work was partially funded by ECOS-Conicyt grant C07U01 and Fondecyt grants 11060312 and 11090201. Technical support from the Instituto Nacional de Hidráulica is gratefully acknowledged. Scientific discussions with professors Eric Barthélemy and Hervé Michallet from the Laboratoire des Écoulements Géophysiques de Grenoble in France, have also greatly improved the quality of this work, and we sincerely thank them.

\section{REFERENCES}

T.E. Baldock. Long wave generation by the shoaling and breaking of transient wave groups on a beach. Proc. R. Soc. London, A, 462: 1853-1876, 2006.

F. Biésel. Équations générales au second ordre de la houle irrégulière. Houille Blanche, pages 372-376, 1952.

R. Cienfuegos, E. Barthélemy, and P. Bonneton. A fourth order compact finite volume scheme for fully nonlinear and weakly dispersive Boussinesq-type equations. Part I : model development and analysis. Int. J. Num. Meth. Fluids, 51 (11): 1217-1253, 2006.

R. Cienfuegos, E. Barthélemy, and P. Bonneton. A fourth order compact finite volume scheme for fully nonlinear and weakly dispersive Boussinesq-type equations. Part II : boundary conditions and validation. Int. J. Num. Meth. Fluids, 53 (9): 1423-1455, 2007.

R. Cienfuegos, E. Barthélemy, and P. Bonneton. A wave-breaking model for Boussinesq-type equations including roller effects in the mass conservation equation. J. Waterw. Port Coastal Oc. Eng., 136: 10-26, 2010.

T. G. Drake and J. Calantoni. Discrete particle model for sheet flow sediment transport in the nearshore. J. Geophys. Res., 106 (15): 19859-19868, 2001.

S. Hibberd and D.H. Peregrine. Surf and run-up on a beach: a uniform bore. J. Fluid Mech., 95: 323345, 1979.

F. Hoefel and S. Elgar. Wave-induced sediment transport and sandbar migration. Science, 299: 1885, 2003.

T.T. Janssen, J.A. Battjes, and A.R. Van Dongeren. Long waves induced by short-wave groups over a sloping bottom. J. Geophys. Res., 108 (C8): 3252, 2003. 10.1029/2002JC001515.

T.B. Johannessen and C. Swan. On the nonlinear dynamics of wave groups produced by the focusing of surface-water waves. Proc. Roy. Soc. London, A (459): 1021-152, 2003.

H. Karunarathna, A. Chadwick, and J. Lawrence. Numerical experiments of swash oscillations on steep and gentle beaches. Coastal Eng., 52: 497-511, 2005.

J.H. List. A model for the generation of two dimensional surf beat. J. Geophys. Res., 97: 5623-5635, 1992. 
M.S. Longuet-Higgins and R.W. Stewart. Radiation stress and mass transport in gravity waves, with application to 'surf beat'. J. Fluid Mech., 13: 481-504, 1962.

P.J. Lynett, T.R. Wu, and P.L.F. Liu. Modeling wave runup with depth-integrated equations. Coastal Eng., 46: 89-107, 2002.

P.A. Madsen, O.R. Sørensen, and H.A. Schäffer. Surf zone dynamics simulated by a Boussinesq-type model : Part I. Model description and cross-shore motion of regular waves. Coastal Eng., 32: 255-288, 1997.

E. Mignot and R. Cienfuegos. On the application of a Boussinesq model to river flows including shocks. Coastal Eng., 56: 23-31, 2009.

K. Nadaoka, M. Hino, and Y. Koyano. Structure of turbulent flow field under breaking waves in the surf zone. J. Fluid Mech., 204: 359-387, 1989.

H.A. Schäffer. Second-order wavemaker theory for irregular waves. Ocean Eng., 23: 47-88, 1996.

G. Symonds, A.J. Huntley, and A.J. Bowen. Two-dimensional surf-beat: long-wave generation by a time-varying breakpoint. J. Geophys. Res., 87: 492-498, 1982. 